\title{
Prioritizing Design Candidates in Adaptable Design Using the Grey Relational Analysis Approach
}

\author{
Y. Li, D. Xue and P. Gu \\ Department of Mechanical and Manufacturing Engineering \\ University of Calgary \\ Calgary, Alberta, Canada T2N 1N4 \\ liy@ucalgary.ca
}

\begin{abstract}
A new method to evaluate design candidates in adaptable design is introduced in this research using the grey relational analysis approach. In this method, the functions of different adaptable design candidates are classified into fundamental functions and adaptable (optional) functions. These design candidates are evaluated by different life-cycle evaluation measures including adaptability of design, part and assembly costs of manufacturing, operationability by customers, etc. The grey analysis approach is employed in this research to integrate the different evaluation measures for prioritizing different design candidates with different fundamental and adaptable functions. A case study is given to demonstrate the effectiveness of the introduced approach.
\end{abstract}

\section{Introduction}

Adaptable design is a new design paradigm with both economical and environmental benefits [1]. The underlying philosophies of adaptable design are the ability of product to adapt to new requirements and the reuse of a product and design when requirements are changed. Adaptable design is conducted through the replacement of multiple products by a single adaptable product with a set of add-on accessories and/or attachments [1]. Since new functions can be adapted easily with an adaptable design, the costs for achieving these new functions from an adaptable design are usually lower than the costs of completely new designs. In addition, the life-span of a product with adaptable functions is usually longer than the life-span of a product without adaptable functions, since old products can be upgraded with new functions.

A guideline on achieving product adaptability through the adaptable design process was introduced in our previous research [2]. By following this guideline, the design engineers may achieve different design candidates, which all are adaptable and fulfill the design requirements. Therefore, evaluation of these design candidates is required.

Adaptability of design is an evaluation measure that considers design functions and the upgrade of design functions. Other evaluation measures in different lifecycle aspects of products - such as part and assembly costs in manufacturing, ease of operation by customers, etc. - also play important roles for improving competitiveness of products in the market place. Therefore, evaluation of the design candidates considering all these different life-cycle aspects has to be conducted in adaptable design.

Design candidate selection through evaluation of these candidates considering different evaluation measures is a typical multiple criteria decision-making problem. The key issue in solving this problem is to convert the different evaluation measures with different units into comparable evaluation indices. In this research, the grey relational analysis in the grey theory [3] has been employed. The grey relational analysis has been demonstrated as a simple and effective approach for analyzing relationships among different decision parameters (e.g., performance and costs) in multiple criteria decision-making problems [4-7].

The rest of this paper is organized as follows. Section 2 discusses adaptable design and evaluation of design candidates in adaptable design. Section 3 introduces the method to prioritize different design candidates in adaptable design using the grey relational analysis approach. Section 4 gives an example to prioritize different food processing devices 
considering adaptability of design, part and assembly costs of manufacturing, and operationability by customers.

\section{Adaptable Design and Evaluation of Design Candidates in Adaptable Design}

\subsection{Adaptable Design and Adaptability}

The architecture of an adaptable product is designed for achieving adaptability of product to prevent the changes in one place from propagating into the rest of the product. Such product architecture is called "segregated architecture" in adaptable design [8]. The design candidates generated following the guideline of adaptable design process can embody the characteristics of segregated architecture. But different design candidates may have different segregated architectures. Due to the different architectures in diverse design candidates, the physical modules that carry out distinct functions and the interfaces among different modules are usually different [9]. Therefore, evaluation of the different design candidates with different architectures is required for selecting the best design candidate.

Adaptability of an adaptable design can be evaluated by comparing the efforts in adapting an existing product to a new one with the required additional functions with the efforts to directly create a new product with the same required functions [1].

In this research, functions of an adaptable design are classified into two categories: fundamental functions and adaptable (optional) functions. For example, a computer with an AGP slot is a typical adaptable design. When a computer is sold without the AGP graphics card, the functions of the computer are the fundamental functions, while the function of the AGP graphics card is the adaptable function. When a computer with an AGP graphics card is sold, the function of the AGP graphics card is also a fundamental function.

Suppose the probability to adapt the current product with an adaptable function $T p_{i}$ (i.e., the i-th target adaptation) in the future is defined as $\operatorname{Pr}\left(T p_{i}\right)$, the effort to adapt the current product to the new adaptable function $T p_{i}$ is described by $\operatorname{Inf}(S I \rightarrow A S 2)$, and, the effort to create a completely new product with the same function is described by $\operatorname{Inf} f_{(Z E R O \rightarrow I S 2)}$. The adaptability factor, $A F\left(T p_{i}\right)$, is then defined by:

$$
A F\left(T p_{i}\right)=1-\frac{\operatorname{Inf} f_{(S 1 \rightarrow A S 2)}}{\operatorname{Inf} f_{(Z E R O \rightarrow I S 2)}}, 0 \leq A F\left(T p_{i}\right) \leq 1(1)
$$

When all $n$ possible adaptable functions are considered, the adaptability of the existing product is defined by:

$$
A(P)=\sum_{i=1}^{n}\left[\operatorname{Pr}\left(T p_{i}\right) A F\left(T p_{i}\right)\right]
$$

In this method, the costs are usually selected to describe the efforts for adapting an existing product or for creating a new product. In Equation (1), the efforts for adapting an existing product or for creating a new product are defined by:

S1: $\quad$ the physical state of the existing product

$A S 2$ : the actual state with the additional adaptable function

ZERO: the state to design a new product from scratch

IS2: the state with only the required adaptable function

From Equation (1), when the effort for adapting an existing product is greater than the effort for creating a new product, the adaptability factor is calculated as 0 . In this case, no adaptation is required. When the effort for adapting an existing product is smaller than the effort for creating a new product, the adaptability factor is calculated as a value between 0 and 1 . In this case, adaptation of the existing product should be considered. When no extra effort is required to achieve the adaptable function from the existing product, the adaptability factor of this product is 1 .

\subsection{Evaluation of Design Candidates in Adaptable Design}

Adaptability is an evaluation measure from the design perspective that considers functions and upgrade of functions. Since different evaluation measures from different product life-cycle aspects including manufacturing costs, ease of operation, etc. - also influence the competitiveness of the product, evaluation of the adaptable designs considering all relevant life-cycle aspects has to be carried out.

Evaluation of a design considering different evaluation aspects is a typical multiple criteria decision-making problem. Usually these different evaluation measures are modeled with different units representing quantitative and qualitative information of products.

In the case study given in Section 4, four evaluation measures - adaptability of design, part cost and assembly cost of manufacturing, and operationability of customers - are selected for evaluating design 
Table 1. Life-cycle evaluation measures

\begin{tabular}{|c|c|c|c|c|}
\hline Evaluation Measure & Adaptability & Part Cost & Assembly Cost & Operationability \\
\hline Life-cycle Aspect & Design & Manufacturing & Manufacturing & Operation \\
\hline Type of Measure & Quantitative & Quantitative & Quantitative & Qualitative \\
\hline Calculation & Eq. (2) & $\begin{array}{c}\text { Sum of Costs of } \\
\text { All Parts }\end{array}$ & $\begin{array}{c}\text { Sum of Costs for } \\
\text { All Assemblies }\end{array}$ & $\begin{array}{c}\text { Rating on Scale } \\
\text { of } 0-5\end{array}$ \\
\hline
\end{tabular}

candidates. These 4 evaluation measures are summarized in Table 1.

\section{Evaluation of Adaptable Designs Using the Grey Relational Analysis Approach}

Since different evaluation measures of adaptable designs are usually modeled with different units, these measures need to be converted into comparable measures for evaluating different design candidates. In this research, the grey relational analysis method [3] has been employed for integrating the different design evaluation measures into the same environment.

The grey relational analysis is a method in the grey theory that compares evaluation measures quantitatively. This method is used to establish the relations among different evaluation measures by comparing the evaluation measures of a particular design candidate with the best evaluation measures considering all design candidates. The grey relational analysis approach requires less data and less requirements on data distributions, and can analyze many measures to overcome the disadvantages of traditional statistical analysis methods [3].

Evaluation of adaptable designs using the grey relational analysis approach is formulated in the following 6 steps.

1. Establish comparative series and standard series.

The comparative series is an information series with $m$ adaptable design candidates and $n$ evaluation measures described by:

$$
A_{i}=\left(x_{i 1}, x_{i 2}, \cdots, x_{i j}, \cdots, x_{i n}\right), \quad i=1,2,3, \ldots, m
$$

The $m$ adaptable designs and the $n$ evaluation measures of these adaptable designs form an $m$ by $n$ decision matrix, $\mathbf{D}$.

The standard series is a target series modeled by:

$$
A_{0}=\left(x_{01}, x_{02}, \cdots x_{0 j}, \cdots, x_{0 n}\right)
$$

The standard series is composed of the best values for each of all the evaluation measures.

2. Generate the normalized decision matrix $\mathbf{K}$ (dimensionless).

To compare the different evaluation measures, they should be converted into dimensionless evaluation measures using the following 3 steps.

(a) If the evaluation measure is of the larger-thebetter type (e.g., the adaptability), the normalized measure is calculated by:

$$
x_{i j}^{*}=\frac{x_{i j}-\min _{i} x_{i j}}{\max _{i} x_{i j}-\min _{i} x_{i j}}
$$

(b) If the evaluation measure is of the smaller-thebetter type (e.g., the cost), the normalized measure is calculated by:

$$
x_{i j}^{*}=\frac{\max _{i} x_{i j}-x_{i j}}{\max _{i} x_{i j}-\min _{i} x_{i j}}
$$

(c) If the evaluation measure is of the nominal-thebest type, and the target value is selected as $x_{o b j}$, the normalized measure is calculated by:

$$
x_{i j}^{*}=\frac{\left|x_{i j}-x_{o b j}\right|}{\max _{i} x_{i j}-x_{o b j}}
$$

The standard series should be converted into

$$
A_{0}^{*}=\left(x_{01}^{*}, x_{02}^{*}, \cdots, x_{0 j}^{*}, \cdots, x_{0 n}^{*}\right) \text { in the same manner. }
$$

3. Obtain the differences between the comparative series and the standard series.

To discover the degree of the grey relationship, the differences, $\Delta_{0}$, between the normalized decision matrix $\mathbf{K}$ and the normalized standard series $A_{0}{ }^{*}$ are achieved by:

$$
\Delta_{0 i j}=\left|x_{0 j}^{*}-x_{i j}^{*}\right|
$$


4. Calculate the grey relational coefficients.

The grey relational coefficients, $\gamma_{0}$, indicate the contiguous grades between the comparative and standard series. A relational coefficient with higher value represents a closer relationship with the best evaluation measure considering all design candidates. The grey relational coefficient is calculated by:

$$
\gamma_{0 i j}=\frac{\Delta \min +\varsigma \Delta \max }{\Delta_{0 i j}+\varsigma \Delta \max }
$$

Where $\Delta \max$ and $\Delta \min$ are obtained by:

$$
\begin{aligned}
& \Delta \max =\max _{i} \max _{j} \Delta_{0 i j} \\
& \Delta \min =\min _{i} \min _{j} \Delta_{0 i j}
\end{aligned}
$$

$\zeta$ is called a distinguished coefficient, only affecting the relative value without changing the priority. Generally, $\zeta$ is selected as 0.5 .

5. Determine the degree of relation with the standard series.

To achieve the degree of relation with the standard series (i.e., the ideal one), the weighting factors of the evaluation measures must first be decided. These weighting factors can be determined by expert experience or marketing strategies in the firm. The degree of relation with the standard series for a design candidate is calculated by:

$$
\Gamma_{0 i}=\sum_{j=1}^{n}\left[w_{j} \times \gamma_{0 i j}\right]
$$

where $w_{j}$ is the weighting factor for the $j$-th evaluation measure satisfying:

$$
\sum_{j=1}^{n} w_{j}=1
$$

\section{Prioritize the design candidates.}

From the degrees of relation between the comparative and standard series, the design candidates can be prioritized. The design candidate with a larger $\Gamma_{0 \mathrm{i}}$ can better satisfy the requirements that consider different product life-cycle aspects.

This grey relational analysis approach treats each design candidate as a comparative series. The degree of relation between the comparative and standard series for each design candidate is then calculated. At the end, the best design can be selected based on the rankings of all the design candidates.

\section{A Case Study Example}

A case study example is given in this section to illustrate the effectiveness of the introduced method for evaluating adaptable design candidates.

\subsection{Problem Statement}

Various electric-motor-driven based small appliances are used for processing different types of foods in the kitchen. These appliances include stand mixers, hand mixers, blenders and meat grinders, as shown in Figure 1.

These small appliances are used frequently in most homes. Since most of them have similar power driven and control systems, considerable redundancy functions can be found in these food processing appliances.

In this research, we focus on the redesign of a stand mixer to make it adaptable for other functions found in a hand mixer, blender and meat grinder.

\subsection{Two Design Configurations}

By conducting design considering product adaptability with the adaptable design guideline introduced in our previous work [2], two design configurations are identified to fulfill the requirements of a stand mixer with the potential to be adapted to other types of food processing appliances.

The first design configuration, as shown in Figure 2 , provides the functions of a stand mixer together with the functions of a hand mixer, blender and meat grinder. The second design configuration, as shown in Figure 3, provides the functions of a stand mixer together with the functions of a blender.

For the first design configuration, when only the function of the stand mixer is selected as the fundamental function of the product and the functions of a hand mixer, blender and meat grinder are selected as adaptable (optional) functions of the product, the part and assembly costs of this product are usually low, compared with a product with all 4 functions as the fundamental functions. The adaptability of a product with all the 4 functions is high, since no extra efforts are required to achieve any of the required food processing functions.

In this case study, 5 product candidates with different fundamental and adaptable functions are selected considering design configuration 1 . In the same way, 2 product candidates with different fundamental functions and adaptable functions are 


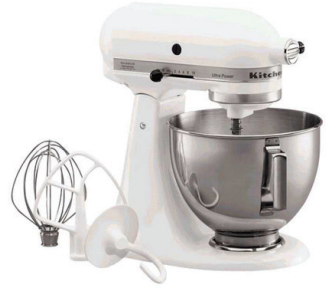

KitchenAid $^{\mathrm{TM}}$ Stand Mixer

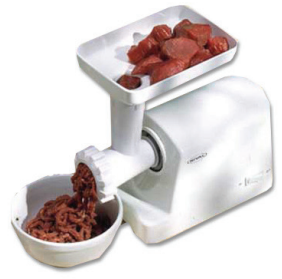

Rival $^{\mathrm{TM}}$ Meat Grinder

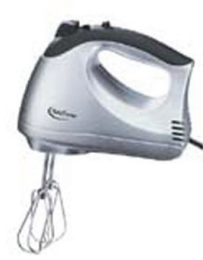

Betty Crocker $^{\mathrm{TM}}$ Hand Mixer
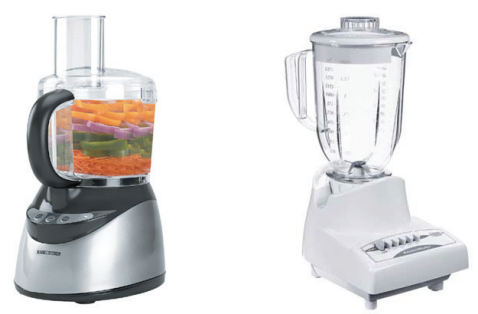

Black \& Decker ${ }^{\mathrm{TM}}$ Food Processor Kitchenworks ${ }^{\mathrm{TM}}$ Blender

Figure 1. Electric-motor-driven based food processing appliances

\section{Basic Function: Stand Mixer}

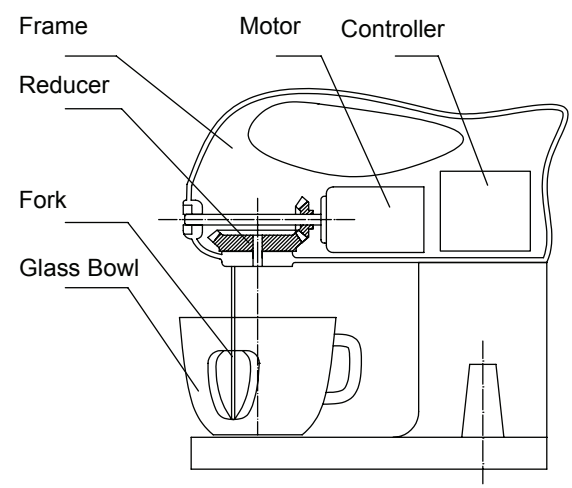

B: Blender

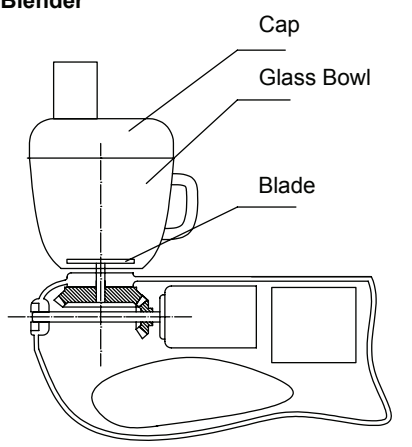

Extra Functions After Adaption

A: Meat Grinder

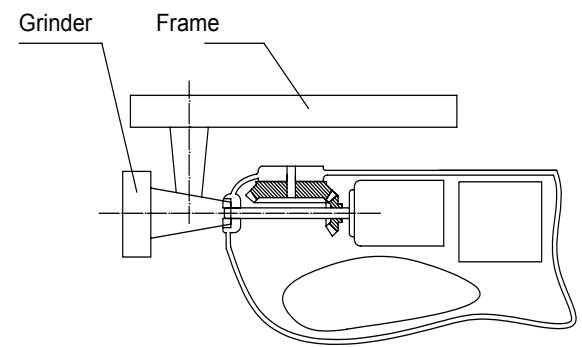

C: Hand Mixer

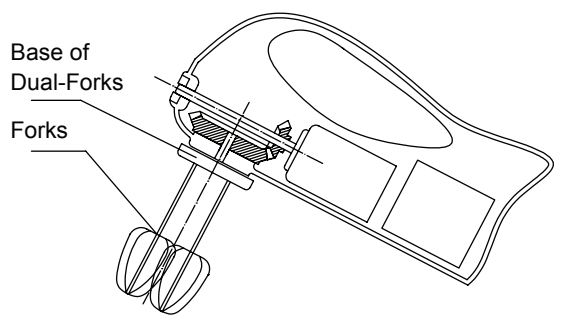

Figure 2. Design configuration 1

selected considering design configuration 2 . These 7 product candidates are summarized in Table 2.

\subsection{Four Measures for Evaluating the Adaptable Designs}

In this work, adaptability, total part cost, total assembly cost, and operationability are selected as the evaluation measures considering design, manufacturing and operation life-cycle aspects of the products.

\section{Adaptabilities of Products}

Adaptability of a product represents its capability of being adapted with additional functions, which are usually initiated by changes in market demand, customer preferences, the operating environment of the product, and the reuse of components of a product after it retires [1]. 

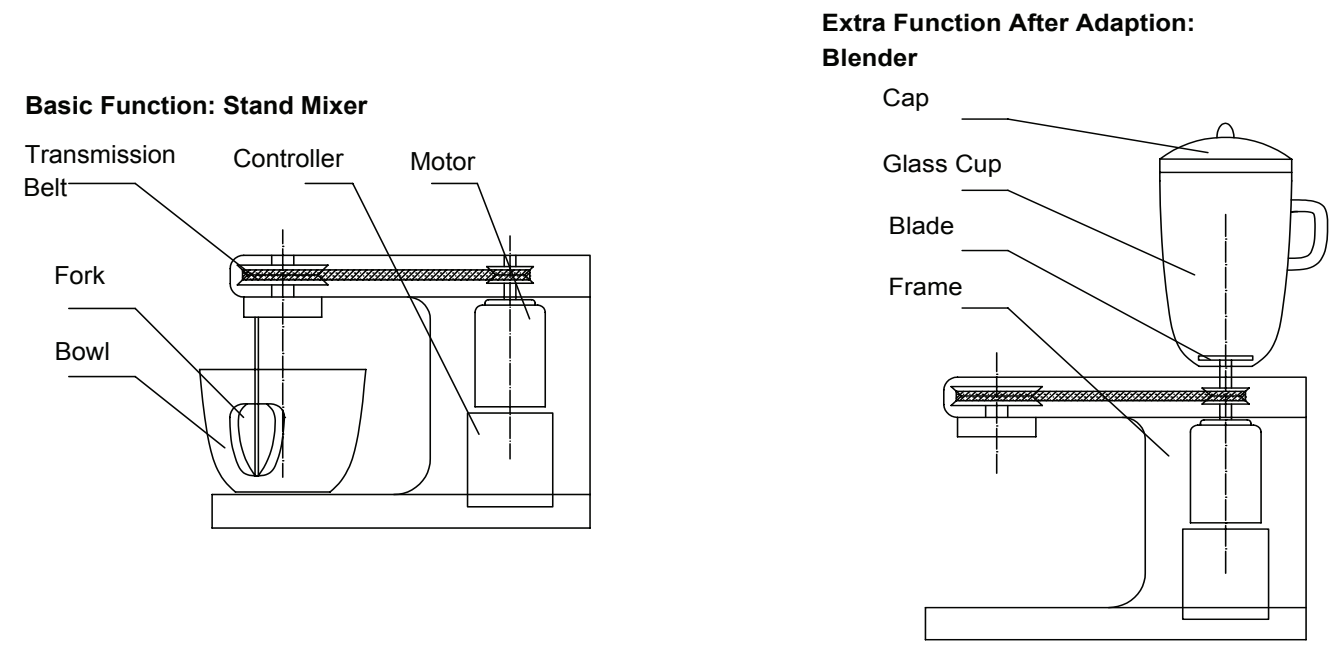

Figure 3. Design configuration 2

Table 2. Product candidates with different fundamental and adaptable functions

\begin{tabular}{|c|c|c|c|c|c|}
\hline Design Configuration & Candidate & Stand Mixer & Grinder & Blender & Hand Mixer \\
\hline \multirow{4}{*}{$\begin{array}{c}\text { Design } \\
\text { Configuration 1 }\end{array}$} & Candidate 1 & $\checkmark$ & $\bigcirc$ & $\bigcirc$ & $\bigcirc$ \\
\cline { 2 - 6 } & Candidate 2 & $\checkmark$ & $\bigcirc$ & $\checkmark$ & $\bigcirc$ \\
\cline { 2 - 6 } & Candidate 3 & $\checkmark$ & $\bigcirc$ & $\checkmark$ & $\checkmark$ \\
\cline { 2 - 6 } & Candidate 4 & $\checkmark$ & $\checkmark$ & $\checkmark$ & $\bigcirc$ \\
\cline { 2 - 6 } & Candidate 5 & $\checkmark$ & $\checkmark$ & $\checkmark$ & $\checkmark$ \\
\hline \multirow{3}{*}{$\begin{array}{c}\text { Design } \\
\text { Configuration 2 }\end{array}$} & Candidate 6 & $\checkmark$ & $\times$ & $\bigcirc$ & $\times$ \\
\cline { 2 - 6 } & Candidate 7 & $\checkmark$ & $\times$ & $\checkmark$ & $\times$ \\
\hline
\end{tabular}

Note: $\checkmark$ indicates the function is a fundamental function in the candidate.

$O$ indicates the function is an adaptable function in the candidate.

$X$ indicates the function cannot be adapted in the candidate.

In this work, the probabilities of using different food processing functions are identified as:

$$
\begin{aligned}
& \operatorname{Pr}(\text { Stand Mixer })=100 \% \\
& \operatorname{Pr}(\text { Grinder })=20 \% \\
& \operatorname{Pr}(\text { Blender })=100 \% \\
& \operatorname{Pr}(\text { Hand Mixer })=50 \%
\end{aligned}
$$

Table 3 shows the costs of parts for design configuration 1 . When the function of the stand mixer is selected as the fundamental function of the product (i.e., candidate 1 in Table 2), the total part cost for this product is then calculated as $\$ 52$. The costs for achieving the functions of a meat grinder, blender and hand mixer are identified as $\$ 10, \$ 8$ and $\$ 7$, respectively. The costs for creating new products of meat grinder, blender and hand mixer are selected as
$\$ 35, \$ 60$ and $\$ 30$, respectively. The adaptability of this candidate is then calculated using Equation (2).

$$
\begin{aligned}
A\left(P_{1}\right) & =100 \% \cdot(1-0)+20 \% \cdot\left(1-\frac{10}{35}\right) \\
& +100 \% \cdot\left(1-\frac{8}{60}\right)+50 \% \cdot\left(1-\frac{7}{30}\right)=2.39
\end{aligned}
$$

For product candidate 2 from Table 2 , the adaptability is calculated by:

$$
\begin{aligned}
A\left(P_{2}\right)= & 100 \% \cdot(1-0)+20 \% \cdot\left(1-\frac{10}{35}\right) \\
& +100 \% \cdot(1-0)+50 \% \cdot\left(1-\frac{7}{30}\right)=2.53
\end{aligned}
$$

The adaptabilities of all other candidates for design configuration 1 can be calculated in the same manner. 
Table 3. The individual part list for design configuration 1

\begin{tabular}{|c|c|c|c|c|c|c|}
\hline No. & Part & Unit Cost (\$) & Stand Mixer & Meat Grinder & Blender & Hand Mixer \\
\hline 1 & Motor with controller & 25 & $\checkmark$ & $\checkmark$ & $\checkmark$ & $\checkmark$ \\
\hline 2 & Reduce gear & 5 & $\checkmark$ & $\checkmark$ & $\checkmark$ & $\checkmark$ \\
\hline 3 & Frame & 10 & $\checkmark$ & $\checkmark$ & $\checkmark$ & $\checkmark$ \\
\hline 4 & Bowl & 10 & $\checkmark$ & & $\checkmark$ & \\
\hline 5 & Blade & 5 & & & $\checkmark$ & \\
\hline 6 & Cap & 3 & & & $\checkmark$ & \\
\hline 7 & Grinder & 10 & & $\checkmark$ & & \\
\hline 8 & Dual-forks base & 5 & & & & $\checkmark$ \\
\hline 9 & Fork & 2 & $\checkmark$ (one) & & & $\checkmark$ (two) \\
\hline
\end{tabular}

Table 4. The individual part list for design configuration 2

\begin{tabular}{|c|l|c|c|c|}
\hline No. & Part & $\begin{array}{c}\text { Unit } \\
\text { Cost (\$) }\end{array}$ & $\begin{array}{c}\text { Stand } \\
\text { Mixer }\end{array}$ & Blender \\
\hline 1 & $\begin{array}{l}\text { Motor with } \\
\text { controller }\end{array}$ & 25 & $\checkmark$ & $\checkmark$ \\
\hline 2 & $\begin{array}{l}\text { Transmission } \\
\text { belt }\end{array}$ & 12 & $\checkmark$ & $\checkmark$ \\
\hline 3 & Frame & 10 & $\checkmark$ & $\checkmark$ \\
\hline 4 & Bowl & 10 & $\checkmark$ & \\
\hline 5 & Glass cup & 10 & & $\checkmark$ \\
\hline 6 & Cap & 3 & & $\checkmark$ \\
\hline 7 & Blade & 5 & & $\checkmark$ \\
\hline 8 & Fork & 2 & $\checkmark$ & \\
\hline
\end{tabular}

Table 4 gives the costs of parts for design configuration 2. For design candidate 6 from Table 2, the cost of the fundamental functions of the stand mixer is calculated as $\$ 59$. The cost to achieve the adaptable function of blender is calculated as $\$ 18$. Suppose the cost to create a new blender product is selected as $\$ 60$, the adaptability of this candidate is then calculated by:

$$
\begin{aligned}
A\left(P_{6}\right) & =100 \% \cdot(1-0)+20 \% \cdot 0 \\
& +100 \% \cdot\left(1-\frac{18}{60}\right)+50 \% \cdot 0=1.7
\end{aligned}
$$

The adaptability of candidate 7 can be achieved in the same way.

The adaptabilities of all the 7 product candidates are summarized in Table 5.

\section{Total Part Costs}

Total part cost for each product candidate is calculated by adding the costs of all parts of which the product is composed. For example, the total part cost of candidate 1 is calculated by:

$$
\begin{aligned}
& C\left(P_{l}\right)=C(\text { Motor with controller })+C(\text { Reduce } \\
& \text { gear })+C(\text { Frame })+C(\text { Bowl })+C(\text { Fork }) \\
& =\$ 25+\$ 5+\$ 10+\$ 10+\$ 2=\$ 52
\end{aligned}
$$

The total part costs of other product candidates are calculated in the same way, as shown in Table 5.

Table 5. Four evaluation measures for seven product candidates

\begin{tabular}{|c|c|c|c|c|}
\hline & Adaptability & Total Part Cost & Assembly Cost & Operationability \\
\hline Candidate 1 & 2.39 & 52 & 10 & 4.5 \\
\hline Candidate 2 & 2.53 & 60 & 10 & 4.5 \\
\hline Candidate 3 & 2.64 & 67 & 10 & 4.5 \\
\hline Candidate 4 & 2.58 & 70 & 13 & 4.5 \\
\hline Candidate 5 & 2.7 & 77 & 13 & 4.5 \\
\hline Candidate 6 & 1.7 & 59 & 15 & 5 \\
\hline Candidate 7 & 2 & 77 & 15 & 5 \\
\hline Type of Value & Quantitative & Quantitative & Quantitative & Qualitative \\
\hline Type of Criteria & $\begin{array}{c}\text { The larger } \\
\text { the better }\end{array}$ & $\begin{array}{c}\text { The smaller } \\
\text { the better }\end{array}$ & $\begin{array}{c}\text { The smaller } \\
\text { the better }\end{array}$ & $\begin{array}{c}\text { The larger } \\
\text { the better }\end{array}$ \\
\hline
\end{tabular}




\section{Assembly Costs}

Assembly cost is the cost for all assembly activities. Different product architectures usually require different assembly costs [10]. In this case study example, the assembly costs of the 7 selected product candidates are given in Table 5.

As more parts are used in a product, the assembly cost usually increases. Since the assembly activities do not require considerable effort in the process of manufacturing, the differences among assembly costs of different candidates are not significant. From Table 5 , we can see product configuration 2 has a higher assembly cost compared with the design configuration 1 , due to its complex architecture.

\section{Operationabilities}

The operationability of products is based on convenience of interface, degree of difficulty to adapt to different functions, and the feelings of customers. Operationabilities are rated on scales between 0 to 5, from totally unsatisfactory to totally satisfactory. The operationabilities of the 7 product candidates are also given in Table 5.

\subsection{Selection of Weighting Factors for Different Evaluation Measures}

The weighting factors of the four different evaluation measures are determined from expert experience and marketing strategies, using statistical methods. These weighting factors are identified as given in Table 6. Since this research focuses on adaptable design, a high weighting factor is selected for the adaptability evaluation measure.

Table 6. Weighting factors of the four evaluation measures

\begin{tabular}{|c|c|}
\hline Evaluation Measure & Weighting Factor \\
\hline Adaptability & $45 \%$ \\
\hline Part Cost & $20 \%$ \\
\hline Assembly Cost & $20 \%$ \\
\hline Operationability & $15 \%$ \\
\hline
\end{tabular}

\subsection{Prioritization of Product Candidates}

Prioritization of the product candidates using the grey relational analysis approach is conducted as follows:
1. Establish the decision matrix $\mathbf{D}$.

$$
D=\left[\begin{array}{cccc}
2.39 & 52 & 10 & 4.5 \\
2.53 & 60 & 10 & 4.5 \\
2.64 & 67 & 10 & 4.5 \\
2.58 & 70 & 13 & 4.5 \\
2.7 & 77 & 13 & 4.5 \\
1.7 & 59 & 15 & 5 \\
2 & 77 & 15 & 5
\end{array}\right]
$$

2. Obtain the standard series.

$$
A_{0}=\left[\begin{array}{llll}
2.7 & 52 & 10 & 5
\end{array}\right]
$$

3. Generate the normalized decision matrix $\mathbf{K}$ and standard series $A_{0}{ }^{*}$.

$K=\left[\begin{array}{llll}0.69 & 1.00 & 1.00 & 0.00 \\ 0.83 & 0.68 & 1.00 & 0.00 \\ 0.94 & 0.40 & 1.00 & 0.00 \\ 0.88 & 0.28 & 0.40 & 0.00 \\ 1.00 & 0.00 & 0.40 & 0.00 \\ 0.00 & 0.72 & 0.00 & 1.00 \\ 0.30 & 0.00 & 0.00 & 1.00\end{array}\right]$

$A_{0}^{*}=\left[\begin{array}{llll}1 & 1 & 1 & 1\end{array}\right]$

4. Calculate the differences between the comparative and standard series.

$$
\begin{aligned}
& \Delta_{0}=\left[\begin{array}{llll}
0.31 & 0.00 & 0.00 & 1.00 \\
0.17 & 0.32 & 0.00 & 1.00 \\
0.06 & 0.60 & 0.00 & 1.00 \\
0.12 & 0.72 & 0.60 & 1.00 \\
0.00 & 1.00 & 0.60 & 1.00 \\
1.00 & 0.28 & 1.00 & 0.00 \\
0.70 & 1.00 & 1.00 & 0.00
\end{array}\right] \\
& \Delta \max =\left[\begin{array}{llll}
1 & 1 & 1 & 1
\end{array}\right] \\
& \Delta \min =\left[\begin{array}{llll}
0 & 0 & 0 & 0
\end{array}\right]
\end{aligned}
$$

5. Determine the grey relational coefficients.

$$
\gamma_{0}=\left[\begin{array}{llll}
0.617 & 1.000 & 1.000 & 0.333 \\
0.746 & 0.610 & 1.000 & 0.333 \\
0.893 & 0.455 & 1.000 & 0.333 \\
0.806 & 0.410 & 0.455 & 0.333 \\
1.000 & 0.333 & 0.455 & 0.333 \\
0.333 & 0.641 & 0.333 & 1.000 \\
0.417 & 0.333 & 0.333 & 1.000
\end{array}\right]
$$


6. Determine the degrees of relations with the standard series.

Using the weighting factors and Equation (12), the degrees of relations for all the 7 product candidates are obtained as:

$$
\begin{aligned}
& \Gamma_{01}=0.728 \\
& \Gamma_{02}=0.708 \\
& \Gamma_{03}=0.743 \\
& \Gamma_{04}=0.586 \\
& \Gamma_{05}=0.658 \\
& \Gamma_{06}=0.495 \\
& \Gamma_{03}=0.471
\end{aligned}
$$

\section{Prioritize the 7 product candidates.}

From the ratings of the product candidates, candidate 3 is selected as the best one, considering all the relevant life-cycle evaluation measures. Compared with candidate 1 , candidate 3 can provide 2 more often-used functions (i.e., blender and hand mixer) with minor additional cost. Compared with candidate 3 , candidate 5 provides only 1 more function (i.e., meat grinder), which is seldom used, with considerable additional cost.

\section{Conclusions}

A new method to evaluate design candidates in adaptable design is introduced in this research using the grey relational analysis approach. In this research, the different design candidates created in adaptable design are evaluated by different life-cycle evaluation measures including adaptability of design, part and assembly costs of manufacturing, operationability of customers, etc. The grey analysis approach is used in this work for prioritizing different design candidates considering different evaluation measures.

The characteristics of this method are summarized as follows:

1. This research introduces a new method for evaluating adaptable designs that considers all relevant life-cycle aspects of the product. In addition to adaptability of design, part and assembly costs of manufacturing, and the operationability by customers are also considered in this work.

2. The grey relational analysis approach is effective for integrating different evaluation measures into the same environment in order to solve multiple criteria decision-making problems. This method can be used for prioritizing different candidates created in adaptable design and for selecting the best one.

\section{Acknowledgements}

This work was supported by the Natural Sciences and Engineering Research Council (NSERC) of Canada and an industrial consortium through the NSERC Design Chair grant.

\section{References}

[1] Gu, P., Hashemian, M., and Nee, A.Y.C, "Adaptable Design”, Annals of CIRP, Vol. 53, No. 2, 2004, pp. 539-557. [2] Li, Y., Xue, D., and Gu, P., "Adaptable Design Process for Product Adaptability: Method and Application", $16^{\text {th }}$ CIRP Design Seminar, Calgary, Alberta, 2006.

[3] Sih, K.C., The Theory of Grey Information Relation, Chun-Hwa Publishing Co., Taipei, 1997.

[4] Chang, Y.C., "The Application of Grey Theory to Purchasing-Decision", Knowledge of Military Supplies, Vol. 94, 1996.

[5] Zhang, J., Wu, D., and Olson, D.L., "The Method of Grey Related Analysis to Multiple Attribute Decision Making Problems with Interval Numbers", Mathematical and Computer Modeling, 2005, pp.1-8.

[6] Wu. H.H., "A Comparative Study of Using Grey Relational Analysis in Multiple Attribute Decision Making Problems", Quality Engineering, Vol. 15, No. 2, 2003, pp. 209-217.

[7] Tosun, N., "Determination of Optimum Parameters for Multi-performance Characteristics in Drilling by Using Grey Relational Analysis", International Journal of Advanced Manufacturing Technology, Vol. 28, 2006, pp. 450-455.

[8] Gu. P., "Adaptable Design with Flexible Interface Systems", Transactions of the SDPS, Vol. 8, No. 3, 2004, pp. 64-74.

[9] Ulrich, K.T., Product Design and Development, McGraw-Hill, New York, 2004.

[10] Boothroyd, G., Dewhurst, P. and Knight, W., Product Design for Manufacture and Assembly, Marcel Dekker, Inc., New York, 1994. 